\title{
Acute Eosinophilic and Neutrophilic Pneumonia following Transarterial Chemoembolization with Drug-Eluting Beads Loaded with Doxorubicin for Hepatocellular Carcinoma: A Case Report
}

\author{
Dimitra Alifakioti $^{\mathrm{a}} \quad$ Cécile Daccord $^{\mathrm{b}}$ Yann Lachenal ${ }^{\mathrm{c}} \quad$ Jean-William Fitting ${ }^{\mathrm{b}}$
}

Departments of a Internal Medicine, ${ }^{\mathrm{b}}$ Respiratory Medicine and ${ }^{\mathrm{C}}$ Radiology and Interventional Radiology,

Lausanne University Hospital, Lausanne, Switzerland

\section{Established Facts}

- Transarterial chemoembolization (TACE) with doxorubicin is established as a treatment for intermediate-stage hepatocellular carcinoma (HCC). The complications of TACE are mainly limited to the liver, spleen and esophagus, but rare cases of acute lung injury have been reported.

\section{Novel Insights}

- This is the first report of a bronchoalveolar lavage performed during acute respiratory distress syndrome following TACE with drug-eluting beads loaded with doxorubicin for HCC. The bronchoalveolar lavage showed a mixed eosinophilic and neutrophilic alveolar inflammation.

\section{Key Words}

Hepatocellular carcinoma · Chemoembolization .

Doxorubicin · Acute lung injury · Eosinophilic pneumonia

\begin{abstract}
At an intermediate or advanced stage, i.e. stage B or C, based on the Barcelona Clinic Liver Cancer classification of hepatocellular carcinoma (HCC), transarterial chemoembolization (TACE) may be offered as a treatment of palliative intent. We report the case of a patient suffering from acute respiratory distress syndrome after TACE with drug-eluting beads loaded with doxorubicin for HCC. To our knowledge, this is the first case described where a bronchoalveolar lavage was per-
\end{abstract}

\section{KARGER}

E-Mail karger@karger.com www.karger.com/res formed, and where significant levels of alveolar eosinophilia and neutrophilia were evident, attributed to a pulmonary toxicity of doxorubicin following liver chemoembolization.

(c) 2014 S. Karger AG, Basel

\section{Introduction}

Hepatocellular carcinoma (HCC) is among the oncological diseases with high prevalence, increased incidence and usually poor prognosis. At the early stage, surgical resection, liver transplantation and percutaneous radiofrequency are potentially curative treatments. When the $\mathrm{HCC}$ is in the intermediate or advanced stage and no cure 


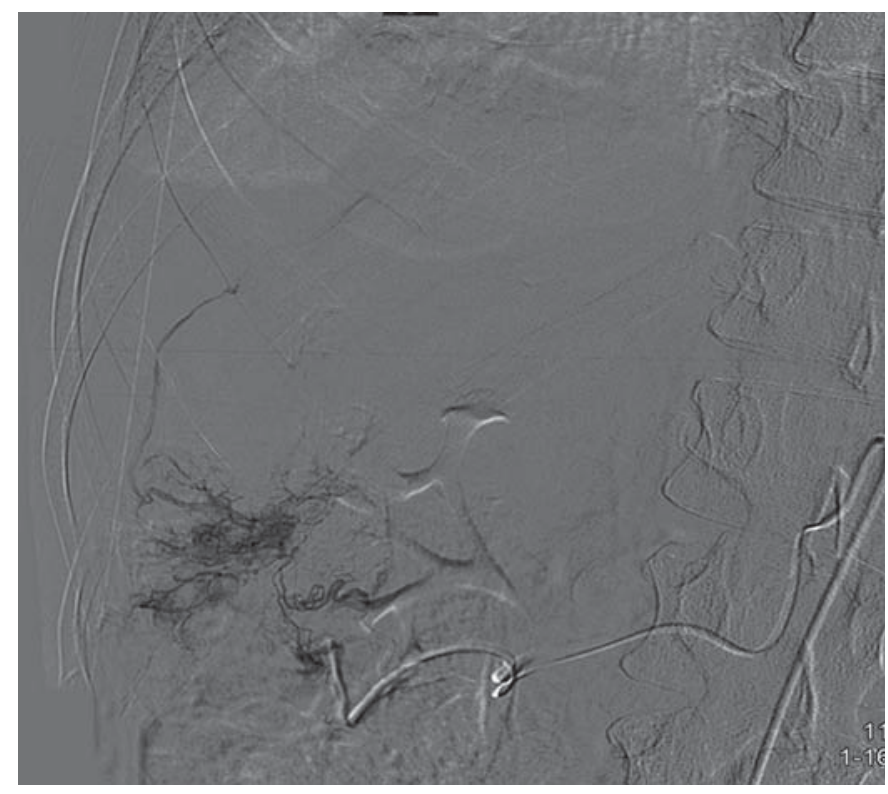

Fig. 1. Hepatic arterial catheterization with a view of the blood vessels supporting the tumor; a subhepatic venous opacity can be seen, compatible with a hepatic arteriovenous shunt.

is possible, a transarterial chemoembolization (TACE) may be performed to inhibit tumor progression. Two techniques are used: conventional TACE or TACE with drug-eluting beads (DEB-TACE) with a diameter of 150$300 \mu \mathrm{m}$ and loaded with a cytotoxic agent, most often doxorubicin. The main side effects are local pain, fever and transient elevation of transaminases and bilirubin. Rarely, the presence of an arteriohepatic shunt may allow the passage of drug-eluting beads with doxorubicin to the pulmonary circulation, causing a toxic pulmonary reaction.

\section{Case Report}

A 48-year-old patient suffering from multifocal HCC (segments II, IV, VI and VIII), previously treated with DEB-TACE loaded with doxorubicin in 2010 and 2013 and by radio-frequency ablation in 2013, was admitted to hospital for the chemoembolization of a new HCC lesion (18 $\mathrm{mm}$ in size) between segments V, VI and VII. As comorbidities, he presented with Child's disease grade $\mathrm{B}$ postalcoholic liver cirrhosis, hepatitis $\mathrm{C}$ and active smoking amounting to 50 pack-years. When admitted, the patient had no respiratory symptoms and pulse oximetry showed that oxygen saturation was 95\%. Pulmonary auscultation was normal and a recent CT scan of the chest did not show any pulmonary parenchyma anomaly. The usual treatment with oxazepam, escitalopram, paracetamol, zolpidem, tramadol, trazodone chlorohydrate and tiotropium bromide was continued.

Eosinophilic Pneumonia after Hepatic Chemoembolization with Doxorubicin
The procedure included a selective catheterization of the right hepatic artery which was feeding the tumoral lesion, followed by a 150 -mg injection of doxorubicin-loaded spherical embolizing microparticles (microbeads) with a diameter of 100-300 $\mu \mathrm{m}$. The arteriography performed initially showed that tumor lesions were fed by multiple abnormal arteries, one of them creating a small arteriohepatic venous shunt through the tumor (fig. 1). This was not considered important enough to give up the chemoembolization, which was performed without any immediate complications.

Forty-eight hours later, the patient became febrile, confused and dyspneic with a new onset of severe hypoxemia $\left(\mathrm{SpO}_{2} 80 \%\right.$ with $\mathrm{FiO}_{2} 1.0$ via facial mask) necessitating his transfer to the intensive care unit. Lung auscultation revealed bilateral basal fine crackles predominant on the left lung. The arterial blood gases under $\mathrm{FiO}_{2} 1.0$ showed: $\mathrm{pH} 7.35, \mathrm{PaCO}_{2} 42.9 \mathrm{~mm} \mathrm{Hg}(5.72 \mathrm{kPa})$ and $\mathrm{PaO}_{2} 61 \mathrm{~mm} \mathrm{Hg}(8.13 \mathrm{kPa})$. The blood tests showed increased white blood cells $(24 \mathrm{~g} / \mathrm{l}), \mathrm{CRP} 62 \mathrm{mg} / \mathrm{l}$, acute renal failure (creatinine $211 \mu \mathrm{mol} / \mathrm{l}$ ), and a significant increase of hepatic cytolysis and cholestasis (ASAT $358 \mathrm{U} / \mathrm{l}$, ALAT $119 \mathrm{U} / \mathrm{l}$ and $\gamma \mathrm{GT} 294 \mathrm{U} / \mathrm{l}$ ). The chest X-ray showed bilateral infiltrates (fig. 2a). An infectious process associated with a possible volume overload was suspected, and a treatment was started with meropenem, diuretic medication and noninvasive ventilation. Forty-eight hours later, a severe hypoxemia persisted with $\mathrm{PaO}_{2} / \mathrm{FiO}_{2}$ ratio of between 80 and $150 \mathrm{~mm}$ Hg. A CT scan of the chest excluded a pulmonary embolism but showed bilateral ground-glass infiltrates with a mosaic pattern and thickening of the interstitial septa (fig. 2b). A bronchoscopy revealed no endobronchial abnormality and the bronchoalveolar lavage revealed an increased number of eosinophils (22\%) and neutrophils (51\%) with a normal lymphocyte count (12\%). Cultures of tracheobronchial secretions and bronchoalveolar fluid remained sterile and the screening for viruses by PCR was negative.

In the absence of administration of any new drug other than doxorubicin, the clinical and biological presentation suggested the pulmonary toxicity of this drug. The patient was administered prednisone $1 \mathrm{mg} / \mathrm{kg}$ per day for 2 weeks, followed by tapering doses to zero over the next 3 weeks. The rapid improvement of his respiratory condition allowed the withdrawal of noninvasive ventilation and a progressive weaning from oxygen supplementation. The chest X-ray confirmed the improvement (fig. 2c).

\section{Discussion}

HCC is a common cancer with increasing incidence and poor prognosis. Most patients are diagnosed when the tumor is already at an advanced stage and is incurable [1]. Palliative therapies such as chemoembolization or radioembolization are available and are being increasingly performed. The vascularization of liver tumors depends on the hepatic arterial supply; chemoembolization aims to cause tumor necrosis by delivering cytotoxic agents directly to the tumor [2].

TACE requires selective catheterization of the tumoral arterial feeders. Two techniques are available with probably equivalent efficacy. Conventional TACE consists of the direct administration of a cytotoxic agent 
Fig. 2. a Chest X-ray obtained $48 \mathrm{~h}$ after chemoembolization showing bilateral pulmonary infiltrates. b Chest CT scan performed 6 days after the chemoembolization showing diffuse ground-glass opacities with a mosaic pattern and thickening of the interstitial septa. c Chest X-ray after 2 weeks of corticosteroid therapy showing regression of the infiltrates.
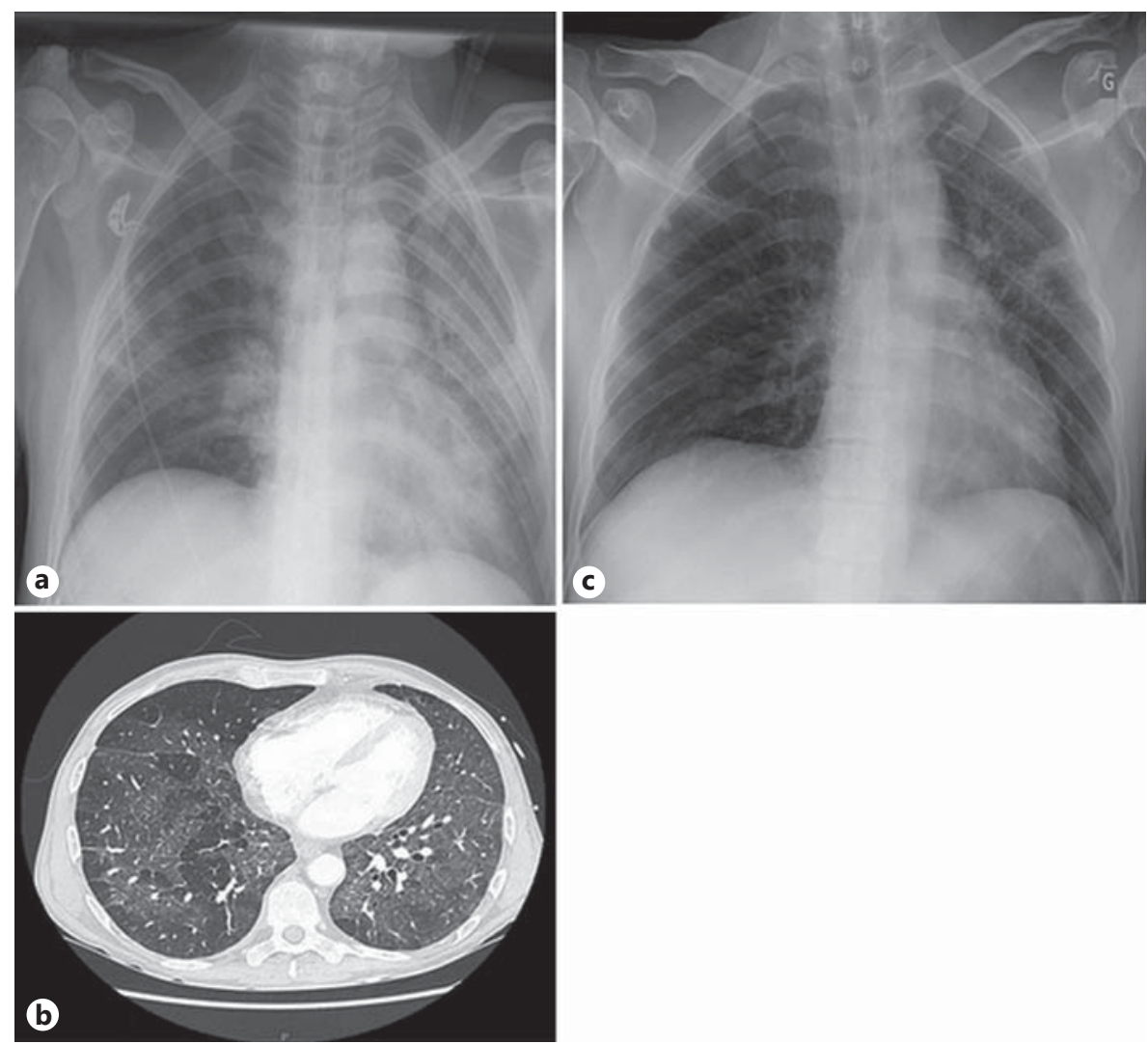

emulsified with lipiodol and followed by microspheres or gelfoam embolization to prolong tumoral exposure to chemotherapy as well as to provoke an ischemia in tumoral lesions. DEB-TACE differs from conventional TACE by the administration of embolic spherical microparticles (100-300 $\mu \mathrm{m}$ in diameter), preloaded with a cytotoxic agent, either doxorubicin in the case of HCC or irinotecan for the refractory liver metastasis of colon carcinoma. DEB-TACE has the advantage of releasing the cytotoxic agent in a progressive and extended way (about a week), thus increasing the effect of the chemotherapy and limiting the systemic toxicity of the treatment [3].

A phase I randomized study (PRECISION V) compared the two techniques and showed a better tolerance and fewer side effects with the DEB-TACE technique in the most fragile patients [1]. The most frequent TACE complications are liver failure, liver abscess, intrahepatic aneurism, acute cholecystitis, splenic infarction, tumor rupture and hemorrhage of gastroesophageal varices [4].

As described in our case, HCC tends to invade hepatic veins and to create arteriohepatic venous shunts. In this circumstance, the TACE, especially with small beads, may permit the embolization of chemoloaded beads in the pulmonary circulation. Using the data of PRECISION $\mathrm{V}$, another publication reported only hepatic, cardiac and gastrointestinal toxicity of the chemoembolization with the doxorubicin-loaded beads [1]. A retrospective analysis in 2009 showed that conventional chemoembolization with lipiodol can cause severe pulmonary damage, typically acute lung injury or acute respiratory distress syndrome [5].

Doxorubicin is a cytotoxic agent used routinely in the treatment of various cancers. The pulmonary side effects of doxorubicin administered systemically include acute lung injury, acute respiratory distress syndrome, secondary organizing pneumonia, pulmonary fibrosis or 'radiation recall pneumonitis' (a rare secondary inflammatory reaction to chemotherapy following radiotherapy). Data on the pulmonary toxicity of DEB-TACE loaded with doxorubicin are scarce. We have identified 2 case reports $[6,7]$ describing the pulmonary toxicity of doxorubicin that manifested in the form of acute lung injury. To our knowledge, our case is the first where bronchoalveolar lavage was performed, and that documents a mixed eosinophilic and neutrophilic alveolar inflammation caused by the pulmonary toxicity of doxorubicin. 
The management of the pulmonary toxicity of doxorubicin mainly addresses symptoms and includes oxygen supplementation associated with ventilator support, depending on the severity of the lung injury. An empirical (i.e. $1 \mathrm{mg} / \mathrm{kg} /$ day) corticosteroid treatment may be initiated, but there is no available data on the adequate dosage and duration of administration for our particular case. By analogy with other drug-induced pneumonitis, some authors suggest high doses of methylprednisone $(1 \mathrm{~g} /$ day for 3 days) in the case of severe respiratory failure and $60 \mathrm{mg}$ i.v. every $6 \mathrm{~h}$ in less severe cases [7].

A whole-body scan with hepatic arterial injection of Tc99m-labeled albumin may be performed before TACE in order to document a possible arteriohepatic venous shunt and thereby limit the risks of the procedure. If a shunt is documented and the procedure is considered to be essential, the use of larger-diameter beads and the adaptation of the drug dose are recommended [7]. One alternative is the occlusion of the shunt in the early stage of the procedure, either by the introduction of a balloon [8] or other embolizing agents (gelfoam particles, steel coils, polyvinyl alcohol particles and embospheres) [4]. In case of a significant shunt $(>20 \%)$, it is preferable to give up the chemoembolization [7].

\section{Conclusion}

Hepatic chemoembolization with drug-eluting beads loaded with doxorubicin is the routinely suggested procedure for the intermediate and advanced stages of HCC. This treatment is mostly well-tolerated and without major complications. However, severe pulmonary toxicity may be caused by the administration of intrahepatic doxorubicin beads in the presence of a hepatic arteriovenous shunt. In our case, severe hypoxemia was associated with a mixed eosinophilic and neutrophilic alveolar inflammation and it regressed after short-term corticosteroid therapy.

\section{References}

$>1$ Vogl TJ, Lammer J, Lencioni R, Malagari K, Watkinson A, Pilleul F, Denys A, Lee C: Liver, gastrointestinal, and cardiac toxicity in intermediate hepatocellular carcinoma treated with PRECISION TACE with drug-eluting beads: results from the PRECISION $\mathrm{V}$ randomized trial. AJR Am J Roentgenol 2011; 197:W562-W570.

-2 Cristina V, Pracht M, Lachenal Y, Adib S, Boubaker A, Prior J, Denys A, Wagner AD, Bize P: Interventional radiology procedures for malignancies of liver treatment: intra-arterial procedures. Rev Med Suisse 2014;10: 1130-1135.
>3 Lammer J, Malagari K, Vogl T, Pilleul F, Denys A, Watkinson A, et al: Prospective randomized study of doxorubicin eluting-bead embolization in the treatment of hepatocellular carcinoma: results of the PRECISION V study. Cardiovasc Intervent Radiol 2010;33: 41-52.

-4 Marelli L, Stigliano R, Triantos C, Senzolo M, Cholongitas E, Davies N, Tibballs J, Meyer T, Patch DW, Burroughs AK: Transarterial therapy for hepatocellular carcinoma: which technique is more effective? A systematic review of cohort and randomized studies. Cardiovasc Intervent Radiol 2007;30:6-25.

$>5$ Wu GC, Perng WC, Chen CW, Chian CF, Peng CK, Su WL: Acute respiratory distress syndrome after transcatheter arterial chemoembolization of hepatocellular carcinomas. Am J Med Sci 2009;338:357-360.
6 Aladdin M, Ilyas M: Chemoembolization of hepatocellular carcinoma with drug-eluting beads complicated by interstitial pneumonitis. Seminars in Interventional Radiology 2011;28:218-221.

$>7$ Khan I, Vasudevan V, Nallagatla S, Arjomand F, Ali R: Acute lung injury following transcatheter hepatic arterial. Lung India 2012;29: 169-172.

8 Lee JH, Won JH, Park SI, Won JY, Lee do Y, Kang BC: Transcatheter arterial chemoembolization of hepatocellular carcinoma with hepatic arteriovenous shunt after temporary balloon occlusion of hepatic Vein. J Vasc Interv Radiol 2007;18:377-382. 